\title{
$X$-Ray Spectral Investigation of Carbon Nanocapsule and Graphite Nanosheet Electronic Structures
}

\author{
Bogdan Ilkiv, ${ }^{1}$ Svitlana Petrovska, ${ }^{1,2}$ Ruslan Sergiienko, ${ }^{2,3}$ Etsuro Shibata, ${ }^{2}$ \\ Takashi Nakamura, ${ }^{2}$ and Yaroslav Zaulychnyy ${ }^{1,4}$ \\ ${ }^{1}$ Frantsevich Institute for Problems of Materials Science, 3 Krzhyzhanivsky Street, Kyiv 03680, Ukraine \\ ${ }^{2}$ Institute of Multidisciplinary Research for Advanced Materials (IMRAM), Tohoku University, Sendai 980-8577, Japan \\ ${ }^{3}$ Physico-Technological Institute of Metals and Alloys NAS of Ukraine, 34/1 Vernadsky Avenue, Kyiv 142, Kyiv 03680, Ukraine \\ ${ }^{4}$ National Technical University of Ukraine "Kyiv Polytechnic Institute", Engineering-Physical Faculty, 35 Polytekchnichna Street, \\ Kyiv 03056, Ukraine
}

Correspondence should be addressed to Ruslan Sergiienko; rsruslan17@gmail.com

Received 5 June 2013; Accepted 9 July 2013

Academic Editors: J. Bai, A. Fidalgo, A. Kajbafvala, and A. Pyatenko

Copyright (C) 2013 Bogdan Ilkiv et al. This is an open access article distributed under the Creative Commons Attribution License, which permits unrestricted use, distribution, and reproduction in any medium, provided the original work is properly cited.

\begin{abstract}
Ultrasoft X-ray emission spectroscopy (USXES) was used to investigate the electronic structures of FePt alloy included carbon nanocapsules (FePt@CNCs) and graphite nanosheets (GNSs). These materials were synthesized by electric plasma discharge in an ultrasonic cavitation field of liquid ethanol. Scanning and transmission electron microscopies were used to study the spatial structures and morphologies. The electronic structure of GNSs was compared with that of reference synthetic graphite powder and high surface area reduced graphene oxide (RGO) sheets with an average thickness of one graphene layer. Investigations of the electronic structures of the FePt@CNC and GNS mixture and GNSs obtained after magnetic separation of the mixture revealed that GNS graphene layers exhibited a smaller curvature than those of carbon nanocapsules, which widened the $\pi$-subband of GNSs due to overlapping of the $p_{\pi}$ orbitals. Thicker GNSs had a narrower $\mathrm{CK}_{\alpha}$ emission band than RGO sheets, which was related to the greater overlapping of $p_{\pi}$ and $\sigma$ orbitals in the more crumpled and corrugated RGO sheets.
\end{abstract}

\section{Introduction}

The growing recent interest in graphene-based nanomaterials, largely because of their electronic structure and resulting interesting properties, has made it necessary to study them in more detail. Graphene Supermarket (https://graphene-supermarket.com/) claims that high surface area graphene structures can be used as reinforcements, conductive additives in composite or coating materials, supports for metallic catalysts, bio and chemical sensors, lowpermeability materials, electrostatic dissipation (ESD) films, and fire retardants. The current two most straightforward and widely used techniques for determining the electronic structures of graphenes are angle-resolved photoemission spectroscopy (ARPES) [1-5] and near-edge X-ray absorption fine structure spectroscopy (NEXAFS) [6-9]. Another direct technique for investigating the electronic structures of solids is X-ray emission spectroscopy, which has been used to study carbon materials: graphite [10], carbon films [11, 12], carbon nanotubes [13], carbon fullerenes [14], and graphene nanoflakes with several graphene layers at the edges [15].

In numerous carbon-based structures, $p$-type electronic states are the main contribution to interatomic interactions [16]. To study the energy distributions of valence Cp electrons in carbon nanomaterials, it is necessary to investigate the $\mathrm{CK}_{\alpha}$ bands. Ultrasoft X-ray emission spectroscopy (USXES) yields comprehensive information about the energy distributions of occupied valence states lower than the Fermi level in carbon materials and is therefore useful for investigating electronic structures.

We report here USXES investigations of graphite nanosheets (GNSs). GNSs were obtained during production of FePt alloy included carbon nanocapsules (FePt@CNCs), using plasma discharge in a liquid ethanol ultrasonic cavitation field [17]. Scanning electron microscopy (SEM) and 


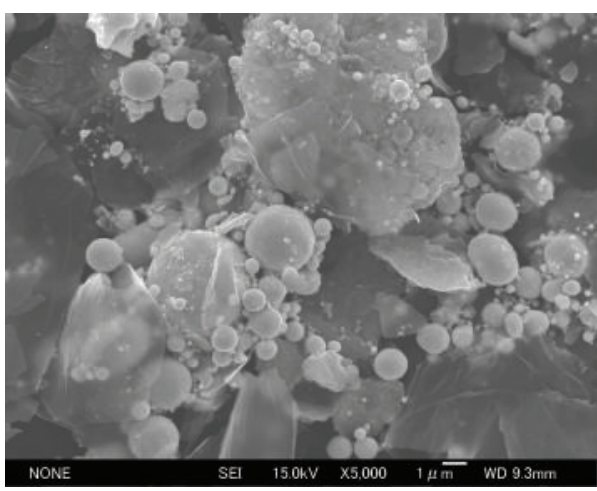

(a)

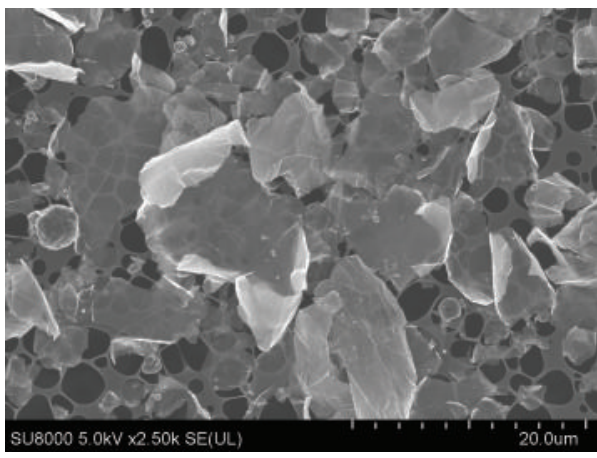

(c)

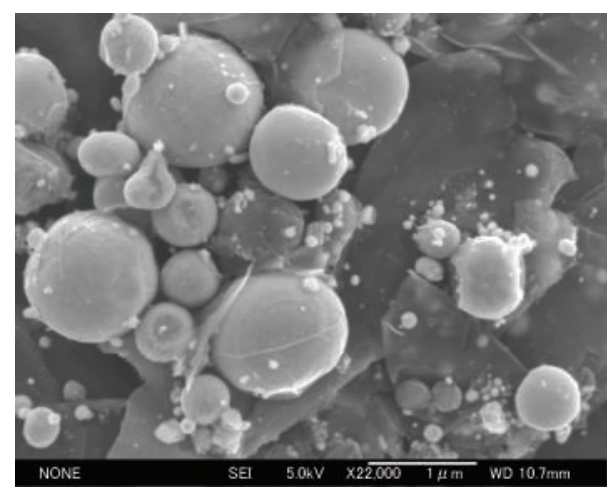

(b)

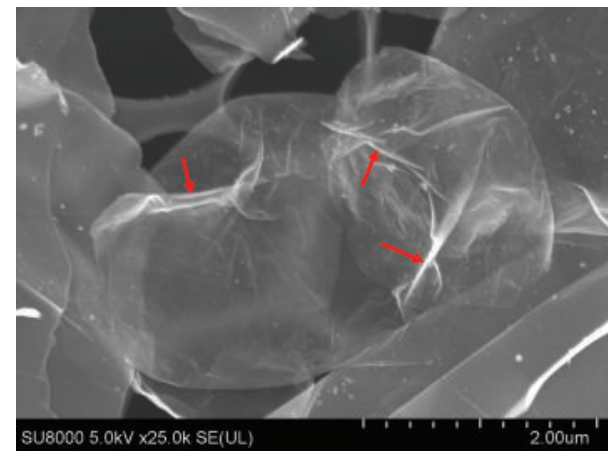

(d)

FIGURE 1: SEM images of carbon powder samples on a carbon TEM microgrid. (a) and (b) FePt@CNC and GNS mixture following hydrogen peroxide oxidation and aqua regia treatment. (c) and (d) Magnetically separated GNSs. Enlarged views of (b) FePt@CNCs and (d) an individual graphite nanosheet with a crumpled structure and folded central regions indicated by arrows.

transmission electron microscopy (TEM) were used to study the GNS and FePt@CNC spatial structures and morphologies. We measured the electronic structures of reference synthetic graphite powder, carbon onions, and high surface area reduced graphene oxide (RGO) sheets, for comparison with that of the synthesized GNSs.

\section{Materials and Methods}

2.1. Materials. Liquid ethanol (99.5\% S-grade), hydrochloric acid (30\%), nitric acid (60\%), and hydrogen peroxide (30\%) were purchased from Wako Pure Chemical Industries, Ltd (Tokyo, Japan). Aqua regia was prepared by mixing one volume of concentrated nitric acid and three volumes of concentrated hydrochloric acid and was used for etching assynthesized carbon powder samples to remove exposed FePt alloy particles. Fe wire $(\varnothing 3,99.9 \%)$ and Pt wire $(\varnothing 2,99.98 \%)$ were used to prepare the 50 at. $\% \mathrm{Fe}$ and 50 at. $\% \mathrm{Pt}(50 \mathrm{Fe} 50 \mathrm{Pt}$ ) alloy in an arc melting furnace. Reference synthetic graphite powder (particle size 1-2 $\mu \mathrm{m}$, ASTM card number 41-1487, CAS number 7782-42-5) was purchased from Sigma-Aldrich Chemical Company, Inc. (St. Louis, MO, USA). RGO with average sheet thickness of one graphene layer was purchased from Graphene Laboratories Inc. (Calverton, NY, USA).
2.2. Preparation and Characterization of GNSs and FePt@CNCs. The detailed synthesis method described previously $[17,18]$ was used to prepare FePt@CNCs and GNSs in a liquid ethanol ultrasonic cavitation field by plasma discharge. The enhanced ultrasound effect is due to the acoustic cavitation phenomena, that is, the rapid formation, growth and the collapse of bubbles in liquid. In an ultrasonic cavitation field, electric conductivity can be improved because of the formation of highly energetic particles (atoms, radicals, and ions) $[19,20]$. A plasma discharge was generated at low voltage, while without ultrasonication, nonconductivity organic liquids required a large breakdown voltage to initiate the plasma discharge. The voltage between the 50Fe50Pt-anode and ultrasonic horn-cathode was constant at $55 \mathrm{~V}$, and the upper electrode current limit was set at $3.0 \mathrm{~A}$ throughout experiments. The plasma flashed from the intermittent decrease in voltage when the current exceeded the power unit limit. This can be further certified by current-voltage characteristic (Figure S-1) in electronic Supplementary Material available online at http://dx.doi.org/10.1155/2013/940545. During experiments, about $10 \mathrm{~mm}$ length of the 50Fe50Pt-anode was consumed by thermal evaporation in the plasma discharge. The carbon powder containing a mixture of FePt@CNCs, GNSs, and amorphous carbon was separated by centrifugation at $5000 \mathrm{rpm}$ for $15 \mathrm{~min}$. Oxidation with $15 \%$ hydrogen 


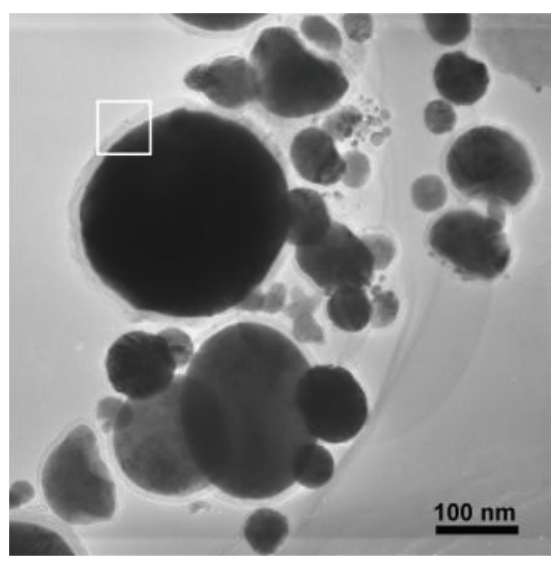

(a)

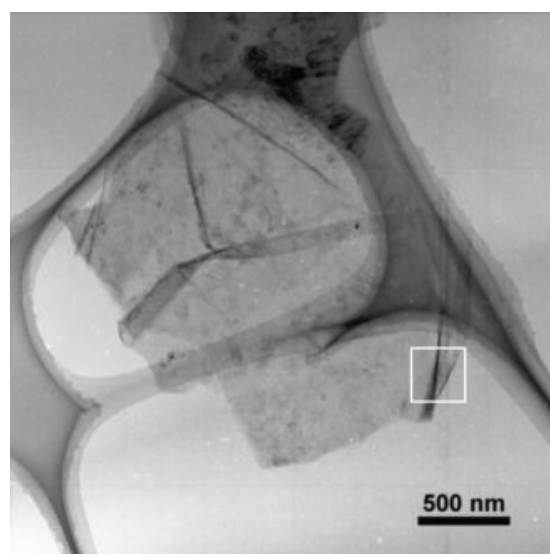

(c)

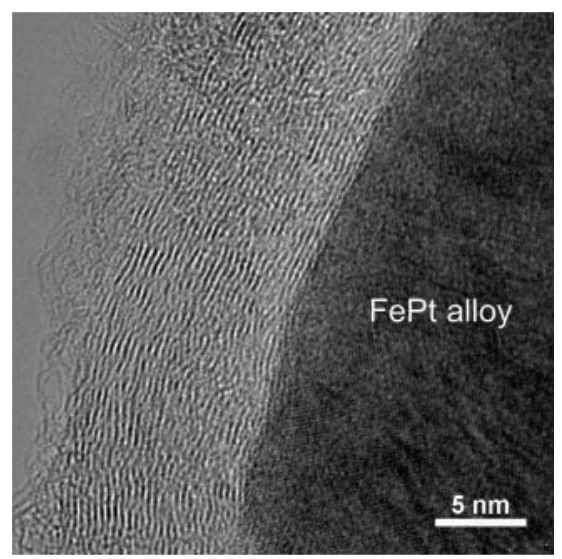

(b)

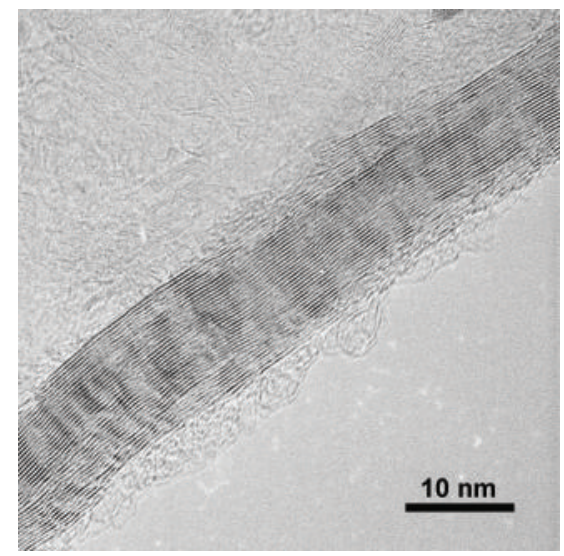

(d)

Figure 2: TEM images of (a) FePt@CNCs and (c) a GNS on TEM microgrids. HR-TEM images of (b) graphene layers in the shell of FePt@CNCs marked in (a) and (d) graphene layers in the curled GNS edge marked in (c).

peroxide solution at $90^{\circ} \mathrm{C}$ for $12 \mathrm{~h}$ removed the amorphous carbon. Additionally, any residual amorphous carbon was separated by carbon powder treatment in an ultrasonic bath with subsequent centrifugation. The remaining mixture of FePt@CNCs and GNSs was etched in aqua regia at $50^{\circ} \mathrm{C}$ for $>12 \mathrm{~h}$ to remove exposed FePt alloy nanoparticles. Finally, FePt@CNCs were removed using a magnetic separator, where the FePt@CNC and GNS dispersion in ethanol was passed between two permanent magnets several times at a rate of $2 \mu \mathrm{m} / \mathrm{s}$, which left the GNSs dispersed in ethanol.

The microstructure and morphology of FePt@CNCs and GNSs were characterized by field emission SEM (FESEM) (Hitachi, SU-8000; JEOL, JSM-7000F), TEM (300 kV, JEOL), and X-ray diffraction (XRD) (Rigaku, RINT-2000) with monochromatic $\mathrm{Cu} K_{\alpha 1}$ radiation source. GNS thickness was measured by dynamic force microscopy (DFM) (NanoNavi/L-trace II).

The ultrasoft X-ray $\mathrm{CK}_{\alpha}\left(K \rightarrow L_{\mathrm{II}, \mathrm{III}}\right.$ transition $)$ emission bands reflecting the energy distribution of the $\mathrm{C} 2 p$ like states in the studied materials were obtained using an RSM-500 spectrometer (SCBXA, Burevestnik, St. Petersburg, Russia). This instrument allowed analysis of spectra in the wavelength region 1-55 nm, where the $\mathrm{CK}_{\alpha}$ emission band is located. An echelette grating with a radius of curvature $6062 \mathrm{~mm}$ and groove density $600 \mathrm{~mm}^{-1}$ was used as a dispersion element. X-ray quanta were registered using a scanning detector on basis of the channel electron multiplier KBL1505 (Dr. Sjuts Optotechnik GmbH, Germany).

Operating conditions of $\mathrm{X}$-ray tubes in experiments were accelerating voltage $U_{a}=5 \mathrm{kV}$ and anode current $I_{a}=$ $2.5 \mathrm{~mA}$. Measurements were carried out under a pressure of $5 \times 10^{-6} \mathrm{~Pa}$ in the spectrometer, obtained using oilfree pumping and freezing out of hydrocarbon vapors by a nitrogen trap near the anode. The spectrometer energy resolution was $0.2 \mathrm{eV}$ in the energy regions corresponding to the position of the $\mathrm{CK}_{\alpha}$ band. The energy positions of the $\mathrm{CK}_{\alpha}$ bands in samples were determined relative to that of $\operatorname{Cr} L_{l}$ line of the pure metal in the second order.

A mixture of FePt@CNCs and GNSs was rubbed directly onto the side of the distended $\mathrm{Cu}$ anode. Separated GNSs were deposited on $\mathrm{Si}$ substrates and pasted onto anode grooves between the adjacent sides with heat- and electricity conductive glue. The anode was cooled using running water at a temperature of $10-15^{\circ} \mathrm{C}$. 


\section{Results and Discussion}

3.1. Morphology of FePt@CNCs and GNSs. A detailed analysis of the structure and morphology of synthesized FePt@CNCs and GNSs has been described elsewhere $[17,18,21]$. The exact mechanism of GNSs formation is currently unclear, and $\mathrm{Pt}$ is probably a crucial factor in the GNS formation process. TEM and SEM observations suggested that GNSs originated from a surface of the FePt@CNCs [21] by an exfoliation mechanism.

Typical SEM images of the mixture of FePt@CNCs and GNSs, freely suspended on TEM carbon grids, are shown in Figures 1(a) and 1(b). Representative SEM images after magnetic separation of the mixture FePt@CNCs and GNSs are shown in Figures 1(c) and 1(d). The graphite nanosheets are irregularly shaped, with an average lateral size of $4-5 \mu \mathrm{m}$ and thickness of $8.8-30 \mathrm{~nm}[17,21]$. A typical SEM image of GNS in Figure 1(d) shows that the nanosheet exhibits good flexibility and is corrugated with many folds, as indicated by arrows. As reported previously [22], corrugation and scrolling are intrinsic to nanosheets and result from vacancies and the introduction of strained pentagon or heptagon rings in the typical hexagonal ring plane.

In agreement with the SEM images (Figures 1(a) and 1(b)), the carbon nanocapsules reach diameter of $\sim 2 \mu \mathrm{m}$. A TEM image of FePt@CNCs with diameters of 10-300 nm is shown in Figure 2(a). The large FePt core is covered by about 30 graphene layers (Figure 2(b)). The thickness of the graphite shell covering the FePt nanoparticles depends on the nanoparticle size and is in the range 1.5-44 $\mathrm{nm}$ [18]. The overall dimensions and thickness of graphite shells covering the FePt nanoparticles appear consistent with evaluated dimensions of the exfoliated GNSs. TEM images of the curled GNS edges could clearly indicate the number of graphene layers, since at a curled edge the sheet is locally parallel to the electron beam (Figures 2(c) and 2(d)). The high-resolution TEM (HRTEM) image of a curled edge in Figure 2(d) shows 30 graphene layers, and a respective GNS thickness of $\sim 10 \mathrm{~nm}$ was calculated at interlayer distance of $0.34 \mathrm{~nm}$.

The XRD carbon peak (002) of GNSs (Figure 3(a)) is clearly divided into two components, at $2 \theta$ of $25.93^{\circ}\left(d_{002}=\right.$ $0.343 \mathrm{~nm})$ and $26.40^{\circ}\left(d_{002}=0.337 \mathrm{~nm}\right)$. This peak is broader than the sharp carbon peak (002) of the reference synthetic graphite powder (Figure $3(\mathrm{~b})$ ). The $d$-spacing $d_{002}=$ $0.343 \mathrm{~nm}$ corresponds to a turbostratic carbon structure; as-synthesized GNSs have a more defective structure than the reference synthetic graphite powder [21]. Reference high surface area RGO sheets exhibit a very broad carbon peak (002) (Figure 3(c)), because the graphene sheets are on average only of one graphene layer thick $(\sim 0.34 \mathrm{~nm})$. RGO sheets are considerably thinner than the GNSs synthesized in this study, and their structure should be more crumpled and flexible. This can be confirmed by SEM and TEM images of RGO sheets (Figure S-2).

3.2. Relationship between Spatial and Electronic Structures. The electronic structures of FePt@CNCs, GNSs, and reference materials (synthetic graphite powder, carbon onions, and high surface area RGO sheets) are compared. Graphite shells covering the FePt cores are similar to carbon onions,
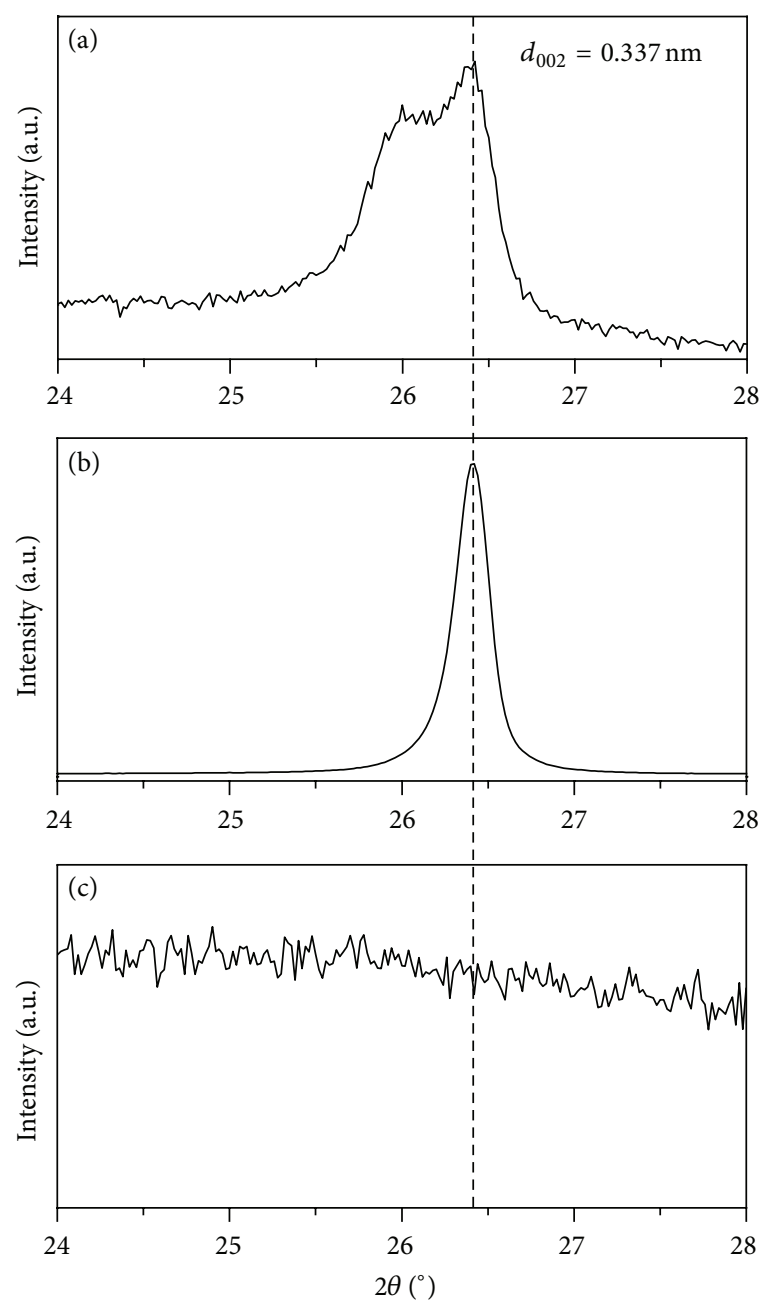

FIGURE 3: XRD carbon peaks (002) of (a) separated GNSs, (b) synthetic graphite powder, and (c) RGO sheets.

but the graphene layers of carbon nanocapsules have a smaller curvature. The diameter of FePt@CNCs ranges from $10 \mathrm{~nm}$ to $2 \mu \mathrm{m}$, while that of carbon onions is $\sim 5 \mathrm{~nm}$. This can be justified by TEM images of a carbon nanocapsule and carbon onions (Figure S-3) [23].

The X-ray emission spectrum of the FePt@CNC and GNS mixture is compared with that of carbon onions (Figure 4). This comparison shows that the fine structures of the $\mathrm{CK}_{\alpha}$ bands are similar, particularly the intensities of the $\pi$ subbands. The smaller surface curvature of the graphene layers in FePt@CNCs causes lower $d$ and $c^{\prime}$ feature intensities. These $d$ and $c^{\prime}$ features represent overlapping of $p_{z}$ and $s p^{n}(2<n<3)$ orbitals, notably a mixture of $\sigma$ and $\pi$ states (Figure 4). The low-energy region of the $\mathrm{CK}_{\alpha}$ band of the FePt@CNC and GNS mixture represents $\sigma$ binding of $s p^{n}$ states and differs from that of the carbon onion spectrum. The locations of the $a$ and $b$ features in the FePt@CNC and GNS mixture are shifted by $0.4-0.5 \mathrm{eV}$ to lower energy. This indicates the higher binding energy of electrons providing the $s p^{n} \sigma$-bonds in the graphene layers of FePt@CNCs, whose curvature is smaller than that of carbon onions. 


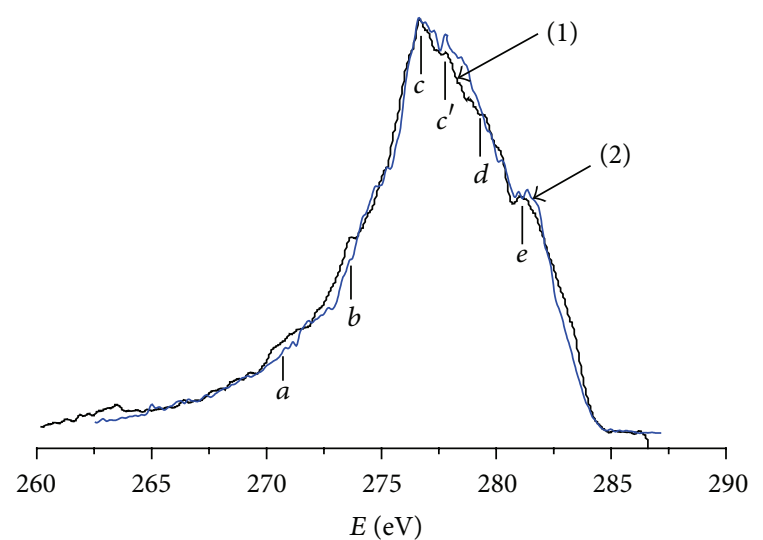

FIGURE 4: $\mathrm{CK}_{\alpha}$ emission bands of (1) FePt@CNC and GNS mixture and (2) and carbon onions.

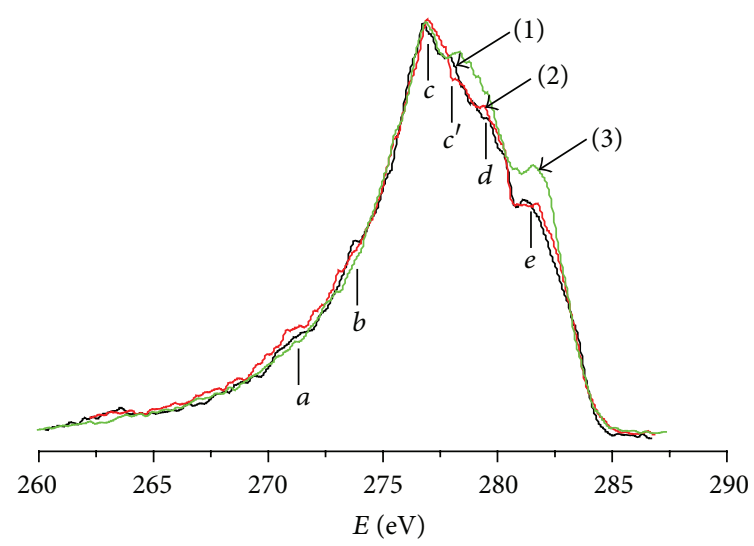

FIGURE 5: CK $K_{\alpha}$ emission bands of (1) FePt@CNC and GNS mixture, (2) magnetically separated GNSs, and (3) synthetic graphite powder.

Magnetically separated GNSs have a lower curvature than that of graphene layers in FePt@CNCs. The overlapping of $p_{\pi}$ orbitals on the GNS surface increases, approaching that of the synthetic graphite powder (Figure 5), and this increase leads to greater splitting of the $\pi$ states. The $\pi$-subband of GNSs approaches that of the synthetic graphite powder $\mathrm{CK}_{\alpha}$ band. However, the intensity of this subband is much lower, probably because of orientational dependence of the $\pi$-subband intensity on the angle of X-ray quanta yield, when electrons move from the $p_{\pi}$ to $1 s$ state [24]. GNSs locate chaotically at the anode relative to the $\mathrm{X}$-ray yield and are not as flat as flakes in synthetic graphite powder.

A $d$ feature is observed in the spectrum of magnetically separated GNSs, as in the spectrum of the FePt@CNC and GNS mixture. This feature corresponds to mixed $\sigma+\pi$ states in synthetic graphite powder, with much higher intensity because of the orientational effect. The $\mathrm{CK}_{\alpha}$ bands in the spectrum obtained from graphene layers in the FePt@CNC and GNS mixture and those of magnetically separated GNSs are largely similar (Figure 5), apart from a widening of the GNS $\pi$ sub-band by $0.3 \mathrm{eV}$. This is related to the decreased GNS curvature compared with that of FePt@CNCs, and the $\pi$-sub-band widens because of overlapping of the $p_{\pi}$

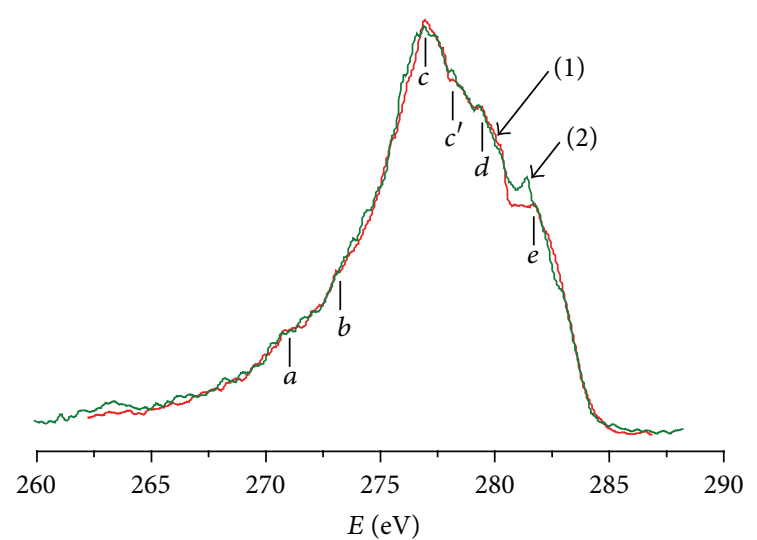

FIGURE 6: $\mathrm{CK}_{\alpha}$ emission bands of (1) magnetically separated GNSs and (2) RGO sheets.

orbitals. The GNSs in this study contain on average $\sim 30$ atomic layers, what is approaching a $\pi$-sub-band near that of synthetic graphite powder (Figure 5). The similar intensity of $\pi$-sub-bands of the FePt@CNC and GNS mixture and GNSs obtained after magnetic separation indicates randomness of GNS location relative to the direction of X-ray quanta emission. The width of the $c$ peak of the $\mathrm{CK}_{\alpha}$ band in the magnetically separated GNSs is reduced, indicating a lower mixing of $\sigma+\pi$ states. Graphene layers in GNSs have a smaller curvature than those in FePt@CNCs, and $p_{\pi}$ orbitals overlap less with $s p^{2}$ orbitals of $\sigma$-type.

The $\mathrm{CK}_{\alpha}$ band of GNSs obtained after magnetic separation of the FePt@CNC and GNS mixture and that of reference graphene sheets obtained by reduction of graphene oxide are largely similar (Figure 6). There are a higher intensity of $e$ sub-band and widening of the $c$ peak in the $\mathrm{CK}_{\alpha}$ band of RGO sheets (Figure 6). The higher intensity of the $\pi$ peak is likely because $\langle 001\rangle$ base planes of the RGO sheets located on the anode parallel to the X-ray yield, leading to a higher intensity of the $e$-sub-band, although they are more crumpled and corrugated than GNSs (see Figure S-2, SEM and TEM images of RGO sheets). Therefore, the yield of X-ray emission is similar to that of synthetic graphite powder. A greater corrugation of RGO sheets leads to greater overlapping of the $p_{\pi}$ orbitals with $\sigma$ orbitals, causing changes of $\sigma$ and $\pi$ states. This leads to widening of the peak of the $\mathrm{CK}_{\alpha}$ band of RGO sheets relative to that of magnetically separated GNSs.

\section{Conclusions}

Investigations of the electronic structures of the FePt@CNC and GNS mixture and GNSs obtained after magnetic separation of the mixture revealed the following:

(1) the binding energy of electrons in $s p^{n}(2<n<$ 3) $\sigma$ bonds in graphene layers of FePt@CNCs is higher than that in carbon onions. This is because of the smaller curvature of the graphite shell in the synthesized carbon nanocapsules,

(2) a smaller mixing of $\sigma+\pi$ states occurs in magnetically separated GNSs compared with the FePt@CNC and 
GNS mixture. This is because synthesized GNSs after magnetic separation have a smaller curvature than carbon nanocapsules, and their $p_{\pi}$ orbitals overlap less with $s p^{2}$ orbitals of $\sigma$ type,

(3) the overlapping of the $p_{\pi}$ orbitals on the surface of magnetically separated GNSs increases and approaches that of synthetic graphite powder,

(4) the thicker synthesized GNSs have a narrower $\mathrm{CK}_{\alpha}$ band than the RGO sheets, whose thickness is only one graphene layer. This is related to the greater overlapping of the $p_{\pi}$ orbitals with $\sigma$ orbitals in the more crumpled and corrugated RGO sheets.

\section{References}

[1] A. Bostwick, J. McChesney, T. Ohta, E. Rotenberg, T. Seyller, and K. Horn, "Experimental studies of the electronic structure of graphene," Progress in Surface Science, vol. 84, no. 11-12, pp. 380-413, 2009.

[2] C. Soldano, A. Mahmood, and E. Dujardin, "Production, properties and potential of graphene," Carbon, vol. 48, no. 8, pp. 2127-2150, 2010.

[3] Z. Luo, J. Shang, S. Lim et al., "Modulating the electronic structures of graphene by controllable hydrogenation," Applied Physics Letters, vol. 97, no. 23, Article ID 233111, pp. 233111-1233111-3, 2010.

[4] M. Kralj, I. Pletikosić, M. Petrović et al., "Graphene on Ir (111) characterized by angle-resolved photoemission," Physical Review B, vol. 84, no. 7, pp. 075427-1-075427-8, 2011.

[5] P. Sutter, M. S. Hybertsen, J. T. Sadowski, and E. Sutter, "Electronic structure of few-layer epitaxial graphene on $\mathrm{Ru}(0001)$," Nano Letters, vol. 9, no. 7, pp. 2654-2660, 2009.

[6] V. Lee, L. Whittaker, C. Jaye, K. M. Baroudi, D. A. Fischer, and S. Banerjee, "Large-area chemically medified graphene films: electrophoretic deposition and characterization by soft X-ray absorption spectroscopy," Chemistry of Materials, vol. 21, no. 16, pp. 3905-3916, 2009.

[7] N. A. Vinogradov, K. Schulte, M. L. Ng et al., "Impact of atomic oxygen on the structure of graphene formed on $\operatorname{Ir}(111)$ and Pt(111)," The Journal of Physical Chemistry C, vol. 115, no. 19, pp. 9568-9577, 2011.

[8] D. Pacilé, M. Papagno, A. F. Rodríguez et al., "Near-edge X-ray absorption fine-structure investigation of graphene," Physical Review Letters, vol. 101, no. 6, pp. 066806-1-066806-4, 2008.

[9] S. Y. Zhou, Ç. Ö. Girit, A. Scholl et al., "Instability of twodimensional graphene: breaking $\mathrm{sp}^{2}$ bonds with soft X-rays," Physical Review B, vol. 80, no. 12, Article ID 121409, 2009.

[10] P. Skytt, P. Glans, D. C. Mancini et al., "Angle-resolved soft-x-ray fluorescence and absorption study of graphite," Physical Review $B$, vol. 50, no. 15, pp. 10457-10461, 1994.

[11] Y. Muramatsu, S. Hirono, S. Umemura et al., "Soft X-ray emission and absorption spectra in the $C K$ region of sputtered amorphous carbon films," Carbon, vol. 39, no. 9, pp. 1403-1407, 2001.

[12] G. Abrasonis, M. Berndt, M. Krause et al., "Soft X-ray absorption and emission spectroscopic investigation of carbon and carbon: transition metal composite films," Journal of Physical Chemistry C, vol. 112, no. 44, pp. 17161-17170, 2008.

[13] Y. V. Zaulichnyi, Y. M. Solonin, S. S. Zvezda, E. V. Prilutskii, O. V. Prilutskii, and E. A. Kats, "Features of the fine structure of the
X-ray CK $\alpha$ emission bands of multiwalled carbon nanotubes," Powder Metallurgy and Metal Ceramics, vol. 45, no. 5-6, pp. 283288, 2006.

[14] E. Z. Kurmaev and S. N. Shamin, "CK $\alpha$ X-ray emission spectra of $\mathrm{C}_{60}$," Physica C, vol. 195, no. 3-4, pp. 352-354, 1992.

[15] C. W. Pao, S. C. Ray, H. M. Tsai et al., "Change of structural behaviors of organo-silane exposed graphene nanoflakes," Journal of Physical Chemistry C, vol. 114, no. 18, pp. 8161-8166, 2010.

[16] V. V. Nemoshkalenko and V. G. Aleshin, Electron Spectroscopy of Crystals, Naukova Dumka, Kiev, Ukraine, 1976.

[17] S. Kim, R. Sergiienko, E. Shibata, Y. Hayasaka, and T. Nakamura, "Production of graphite nanosheets by low-current plasma discharge in liquid ethanol," Materials Transactions, vol. 51, no. 8, pp. 1455-1459, 2010.

[18] R. Sergiienko, S. Kim, E. Shibata, and T. Nakamura, "Structure of Fe-Pt alloy included carbon nanocapsules synthesized by an electric plasma discharge in an ultrasonic cavitation field of liquid ethanol," Journal of Nanoparticle Research, vol. 12, no. 2, pp. 481-491, 2010.

[19] K. S. Suslick, N. C. Eddingsaas, D. J. Flannigan, S. D. Hopkins, and $\mathrm{H} . \mathrm{Xu}$, "Extreme conditions during multibubble cavitation: sonoluminescence as a spectroscopic probe," Ultrasonics Sonochemistry, vol. 18, no. 4, pp. 842-846, 2011.

[20] D. J. Flannigan and K. S. Suslick, "Inertially confined plasma in an imploding bubble," Nature Physics, vol. 6, no. 8, pp. 598-601, 2010.

[21] R. Sergiienko, S. Kim, E. Shibata, Y. Hayasaka, and T. Nakamura, "Structure of graphite nanosheets formed by plasma discharge in liquid ethanol," Powder Metallurgy and Metal Ceramics, vol. 52, no. 5-6, 2013.

[22] J. C. Meyer, A. K. Geim, M. I. Katsnelson, K. S. Novoselov, T. J. Booth, and S. Roth, "The structure of suspended graphene sheets," Nature, vol. 446, no. 7131, pp. 60-63, 2007.

[23] O. O. Mykhaylyk, Y. M. Solonin, D. N. Batchelder, and R. Brydson, "Transformation of nanodiamond into carbon onions: a comparative study by high-resolution transmission electron microscopy, electron energy-loss spectroscopy, X-ray diffraction, small-angle X-ray scattering, and ultraviolet Raman spectroscopy," Journal of Applied Physics, vol. 97, no. 7, pp. 074302-074318, 2005.

[24] W. Burghard, M. Umeno, G. Wiech, and W. Zahorowski, "Xray spectroscopic study of carbon fibres and graphitic carbon," Journal of Physics C, vol. 16, no. 21, pp. 4243-4258, 1983. 

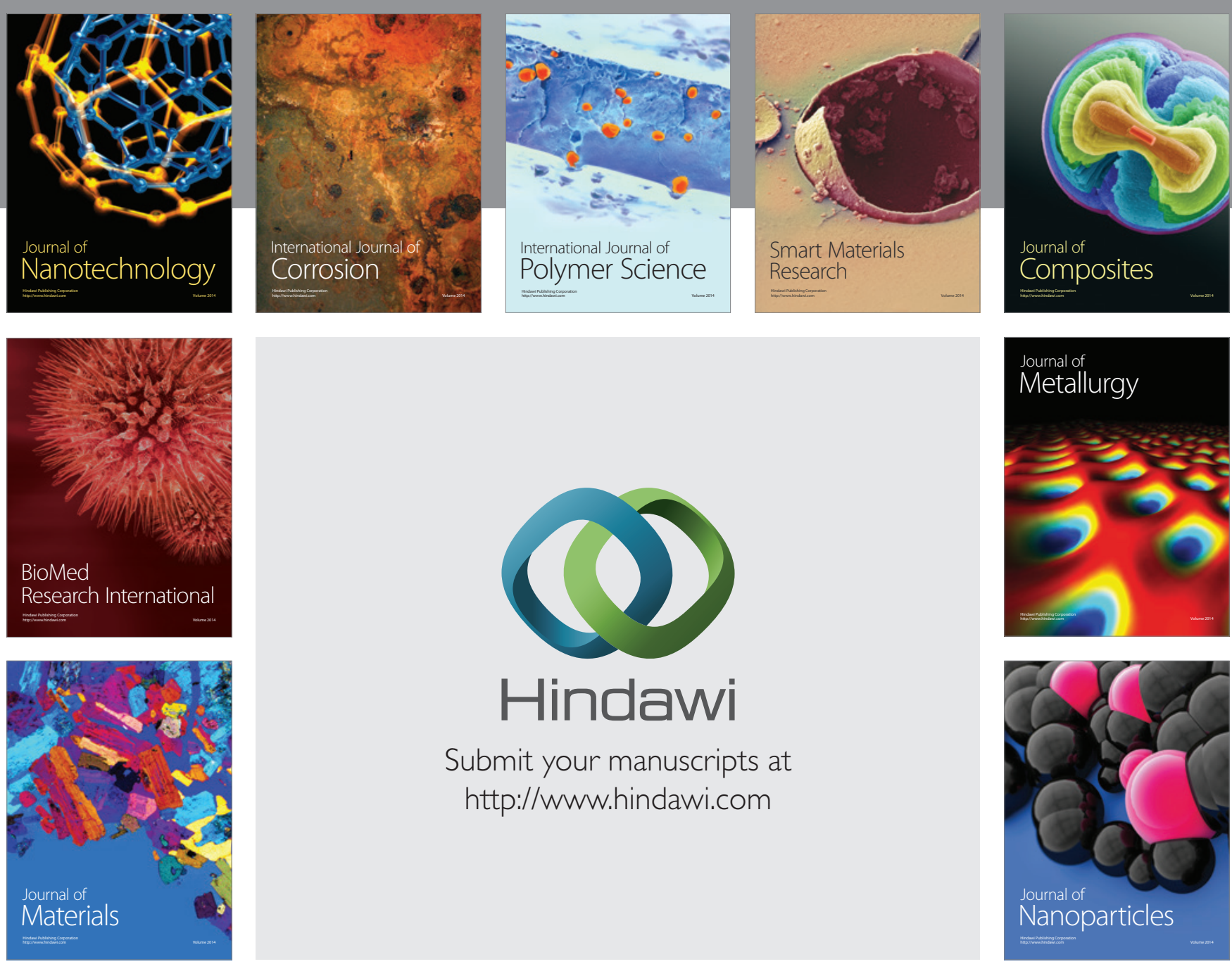

Submit your manuscripts at http://www.hindawi.com
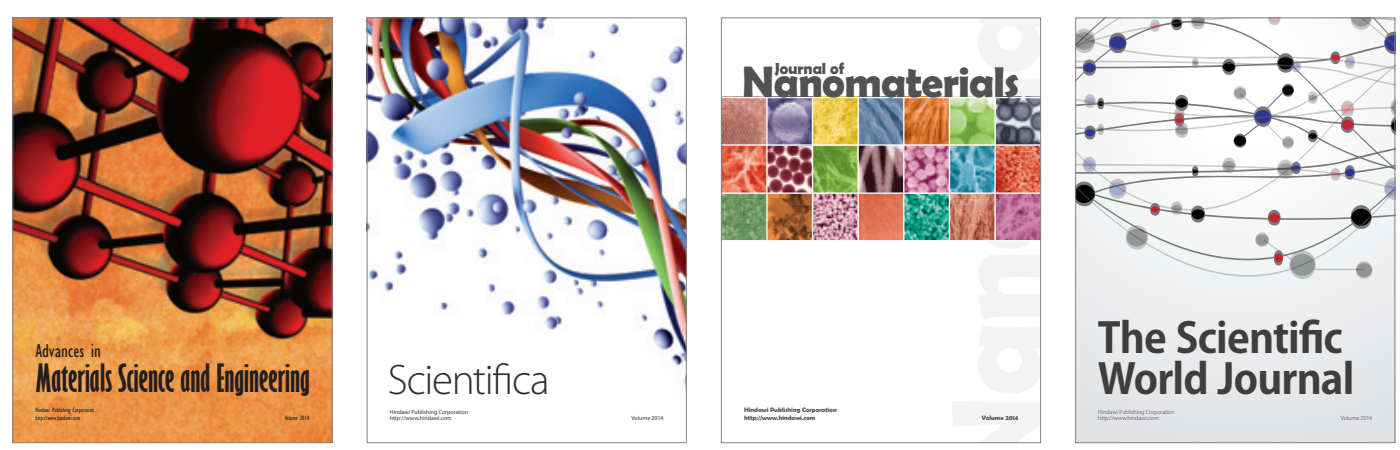

\section{The Scientific World Journal}
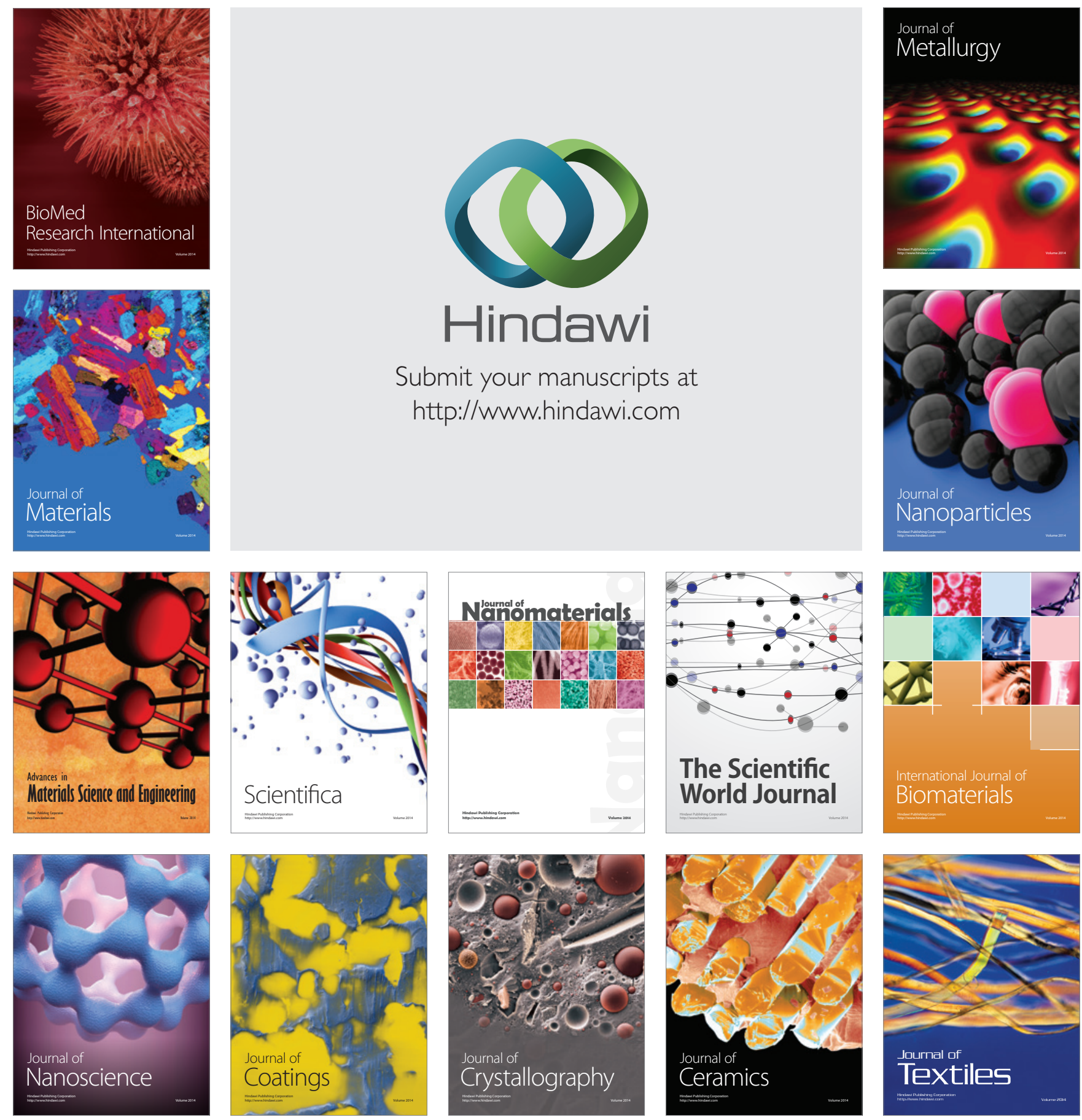